\title{
Early Peripheral Blood Blast Clearance: A New Chemosensitivity Indicator for Acute Myeloid Leukemia
}

\author{
Su Long* \\ Cancer center, the First Hospital, Jilin University
}

*Corresponding author: Su Long, Cancer center, the First Hospital, Jilin University, Tel: +86-0431-88782157, Fax: +86-0431-88782688, E-mail: sulongjdyy@163.com

Citation: Su Long (2014) Early Peripheral Blood Blast Clearance: a New Chemosensitivity Indicator for Acute Myeloid Leukemia. SAJ Cancer Sci 1: 103. doi: 10.18875/2375-6683.1.103

Article history: Received: 12 June 2014, Accepted: 02 September 2015, Published: 04 September 2014

Acute myeloid leukemia (AML) is a highly heterogeneous disease, and cytogenetics and molecular markers play important roles in the diagnosis, treatment, and prognosis of AML. Although cytogenetic karyotypes and molecular mutations are powerful prognostic predictors for outcome of AML patients, indicators of response to initiation chemotherapy have not been identified. In childhood acute lymphoblastic leukemia, a rapid decline of circulating leukemic blasts in response to induction chemotherapy or prednisone is one of the most important prognostic factors, not only for achieving remission but also for long-term survival [1]. Similarly, in patients with AML who have received induction chemotherapy, a rapid decrease in peripheral blood blasts (PBB) is also considered as a favorable indicator of response to chemotherapy [1-4]

The timing of $\mathrm{PBB}$ clearance is critical for complete remission (CR) and survival in patients with AML. Based on a study with 30 AML patients, Gianfaldoni et al. reported that the kinetics of PBB clearance was a predictor of CR [2]. Elliott et al. demonstrated that the time to clearance of PBB during standard induction therapy was a strong predictor of both overall survival (OS) and relapse-free survival (RFS) in a group of 73 AML patients [1]. Quintas-Cardama et al. showed that early clearance of PBB was an important risk factor for the achievement of CR and for OS in 486 uniformly treated AML patients [3]. In 162 patients with AML, Arellano et al. recently reported that early clearance of PBB ( $\leq 6$ days) after induction chemotherapy was a predictor of early blast clearance from the marrow (day 14), CR, RFS, and OS [4]. In this year's European Hematology Association meeting, Gianfaldoni et al. reported that early PBB clearance could predict both CR and event-free survival in 178 AML patients participated into the NILG AML 02/06 clinical trial [5]. Residual PBB count or percentage in initiation chemotherapy was also very important for AML. We observed that PBB percentage on day 7 (D7PBBP), the last day of the " $3+7$ " regimen, of induction therapy, not only could predict $\mathrm{CR}$, but also were associated with OS and RFS in 46 consecutive Chinese patients with AML [6]. Considering the association between PBB clearance and long-term outcomes, Elliott et al. defined three "blast risk groups" as good, intermediate, and poor risk, according to the rate of blast clearance on or before day 3 , on days 4 or 5 , or on day 6 or beyond, respectively [1]. Likewise, we proposed a new risk-adapted stratification system based on D7PBBP [6] (Table 1). According to our observation, patients in good risk group had a high CR rate and good survival, those in intermediate risk group had a high CR rate and intermediate survival, whereas those in high risk group had a low CR rate and poor survival. These findings suggest that consolidation therapy may be important for patients of intermediate risk group, and both early intervention and consolidation therapy should be considered for patients of high risk group [6].

\begin{tabular}{|c|c|c|}
\hline & Elliot's stratification & Our stratification \\
\hline Good risk & PBB clearance at or before day 3 & D7PBBP $<0.43 \%$ \\
\hline Intermediate risk & PBB clearance on days 4 or 5 & $0.43 \% \leq$ D7PBBP $<0.945 \%$ \\
\hline High risk & PBB clearance at or after day 6 & D7PBBP $\geq 0.945 \%$ \\
\hline
\end{tabular}

Table 1: Risk-adapted stratification for AML based on early PBB clearance

As a result, all these findings suggested that early $\mathrm{PBB}$ clearance could be a potential indicator for chemosensitivity in patients with AML. However, large-scale study is still needed to establish the criterion for predicating CR based on PBB clearance, and risk-adapted stratification. 


\section{References}

1. Elliott MA, Litzow MR, Letendre LL, Wolf RC, Hanson CA, et al. (2007) Early peripheral blood blast clearance during induction chemotherapy for acute myeloid leukemia predicts superior relapse-free survival. Blood 110: 4172-4.

2. Gianfaldoni G, Mannelli F, Baccini M, Antonioli E, Leoni F, et al. (2006) Clearance of leukaemic blasts from peripheral blood during standard induction treatment predicts the bone marrow response in acute myeloid leukaemia: a pilot study. Br J Haematol 134: 54-7.

3. Quintas-Cardama A, Kantarjian H, Garcia-Manero G, Mark Brandt BS, Sherry Pierce, et al. (2011) Early clearance of peripheral blood blasts but not white blood cells is a powerful prognostic marker for complete response and overall survival in patients with acute myeloid leukemia (AML) receiving induction chemotherapy. ASH 1553.

4. Arellano M, Pakkala S, Langston A, Tighiouart M, Pan L, et al. (2012) Early clearance of peripheral blood blasts predicts response to induction chemotherapy in acute myeloid leukemia. Cancer 118: 5278-82.

5. Gianfaldoni G, Mannelli F, Intermesoli T, et al. (2014) Early peripheral blast cell clearance assessed by flow cytometry in induction in a novel powerful prognostic indictor in acute myeloid leukemia: a Northern Italy Leukemia Group (NILG) study. EHA 4555.

6. Gao SJ, Tan YH, Liu XH, Su L, Yu P, et al. (2014) The percentage of peripheral blood blasts on day 7 of induction chemotherapy predicts response to therapy and survival in patients with acute myeloid leukemia. Chin Med J (Engl) 127: 290-3. 